\title{
Análisis Psicológico de la Actividad Física en Estudiantes de una Universidad de Bogotá, Colombia
}

\author{
Maria T. Velásquez ${ }^{1}$, Danilo Torres-Neira ${ }^{2}$ y Henry Sánchez-Martínez ${ }^{3}$ \\ ${ }^{1}$ Psicóloga. M. Sc. Educación. Departamento de Psicología. Facultad de \\ Ciencias Humanas Universidad Nacional de Colombia. Teléfono: 2566112. \\ ${ }^{2}$ Psicólogo. Investigador independiente.E-mail: danilo_tn@yahoo.com \\ ${ }^{3}$ Psicólogo. Investigador independiente. E-mail: henrry.sanchez@gmail.com
}

Recibido 31 Mayo 2005/Enviado para Modificación 10 Agosto 2005/Aceptado 27 Septiembre 2005

\section{RESUMEN}

Objetivo Se buscó delimitar y explicar los factores psicológicos inherentes en la no práctica, práctica y mantenimiento de la actividad física en la población estudiantil de la Universidad Nacional de Colombia, en el año 2004.

Métodos Se empleó el modelo del Proceso de Adopción de Precauciones (PAP) para comprender la conducta de la actividad física como proceso y estado, desde el sedentarismo hasta la formación de estilos de vida activos. Se realizó la adaptación del modelo PAP para la explicación de la conducta en cuestión, en donde se sopesaron los factores psicológicos a través de cada una de las etapas de cambio conductual. Se valida la adaptación lograda mediante el diseño y aplicación de un instrumento psicométrico que evalúa las tendencias de práctica, no práctica y mantenimiento de actividad física en estudiantes de la Universidad Nacional de Colombia.

Resultados La aplicación del instrumento devela la influencia y relación de los factores psicológicos que intervienen directamente en las etapas del modelo propuesto.

Conclusiones El modelo es un sólido constructo teórico-metodológico, que da cuenta de forma adecuada de los factores psicológicos que intervienen en la no práctica, práctica y mantenimiento de la actividad física. En este sentido el modelo puede ser utilizado en la creación de una estrategia de intervención en promoción de la salud y prevención de enfermedad a partir de estilos de vida activos. 
Palabras Clave: Actividad motora, promoción de la salud, estilo de vida, calidad de vida (fuente: DeCS, BIREME).

\section{ABSTRACT \\ Psychological analysis of university students' physical activity in Bo- gotá, Colombia}

Objective Determining and explaining inherent psychological features in the non-practice, practice and maintenance of physical activity in the Universidad Nacional de Colombia's students during 2004.

Methods The precaution adoption process (PAP) model was used for understanding physical activity patterns from leading sedentary lifestyles to forming active ones. Psychological features are presented from health psychology and sports psychology viewpoints. The PAP model was adapted for explaining the behaviour in question; psychological features were carefully considered during each stage of behavioural change. The adaptation was validated by designing and applying a psychometric instrument evaluating the tendency of students from the $U$. Nacional to practice, not practice and maintain their physical activity.

Results Applying the instrument revealed the influence and relationship of the psychological features directly involved in the stages of the proposed model.

Conclusions The model was a solid theoretical and methodological construct, suitably expressing all psychologist features intervening in the nonpractice, practice and consolidation of physical activity. The model can thus be used in creating an intervention strategy related to promoting health and preventing disease through encouraging active lifestyles.

Key Words: Motor activity, health promotion, life style, quality of life (source: $\mathrm{MeSH}, \mathrm{NLM}$ ).

$\mathrm{E}$ n la sociedad actual se puede evidenciar una constante disminución de la práctica de actividad física debido a varios factores dentro de los cuales se encuentra la sistematización o automatización de los procesos productivos y de la vida cotidiana. Las personas se encuentran muy interesadas en reducir el esfuerzo físico al realizar todas las tareas que se presentan diariamente. Por tal razón en las últimas décadas se ha desarrollado un importante interés en el estudio de cómo la falta de práctica de actividad física (sedentarismo) afecta la salud y el bienestar de la población (1-3).

Cálculos realizados por el National Center for Chronic Disease Prevention and Health Promotion (1-3) reportan que el $27 \%$ de la población joven del continente americano es suficientemente activa, quedando un $73 \%$ de población que no practica actividad física o deporte regularmente. Debido a 
esta baja tasa de práctica en este segmento poblacional, surge la inquietud por profundizar el análisis de este fenómeno en una población mucho más particular, los estudiantes universitarios. Los estudios más destacados que se han desarrollado acerca de la relación entre actividad física y jóvenes universitarios se refieren a las influencias que los estilos de vida activos pueden tener en la salud. En cuanto a la influencia positiva de los estilos de vida activos en los jóvenes universitarios, se encuentran beneficios psicológicos (4), prevención de enfermedades (5-11) y bienestar general (1213).

Para desarrollar el estudio en este grupo de edad, se abordó la población de la Universidad Nacional de Colombia. Esta población se desenvuelve en un ambiente con rica infraestructura para la práctica de actividad física, y con políticas que buscan promover la práctica de actividad física por parte de la Universidad (14). A pesar de estas condiciones, la población universitaria no hace el uso ideal de éstas. Así, la Dirección de Bienestar Universitario (14), indica que solamente el $12 \%$ de estudiantes hacen uso de estas herramientas, existiendo aproximadamente un $88 \%$ de estudiantes de los cuales no se tiene información en sus hábitos de práctica de actividad física.

Se evidencia entonces que el problema de la práctica de actividad física en los estudiantes va más allá de la infraestructura o políticas institucionales, por obvias razones. Se parte entonces del análisis conductal y psicológico de la problemática. Para tal fin, se requiere de un modelo explicativo que articule conceptos tomados desde la psicología de la salud que dan cuenta de los procesos de adquisición o modificación de estilos de vida, y desde la psicología del deporte referentes a la compresión de los procesos propios de la práctica de actividad física.

Modelos de comprensión de la actividad física

Dentro de la promoción de la salud a través de la psicología, se han desarrollado varios modelos de gran importancia (15). Un grupo de modelos que más influencia ha tenido en los estudios de modificación y creación de estilos de vida saludables son los modelos de etapas (16). Estos modelos contemplan los diferentes procesos y niveles que darían lugar a un cambio conductual. Sugieren que las personas tienen conductas, pensamientos y emociones cualitativamente diferentes en cada nivel del proceso de adopción de estilos de vida, existiendo barreras de un nivel a otro. De igual manera proponen que las intervenciones hacia la modificación conductual varían de un nivel a otro. 
Dentro de los modelos de etapas el modelo del PAP ha sido el más utilizado para la promoción de la salud, en diferentes ámbitos y con diferentes objetivos. En Colombia, Flórez ha realizado su adaptación a las condiciones de la población y ha desarrollado varios procesos de intervención hacia la prevención de consumo de sustancias psicoactivas y promoción en salud sexual y reproductiva (17-18). Debido a las ventajas teórico-prácticas de los modelos de etapas, y a la adaptación de modelo del PAP a la población colombiana en promoción de la salud y prevención de enfermedades, se parte del PAP como piedra angular para la comprensión de los procesos que se desarrollan en la práctica de actividad física.

Modelo del PAP adaptado a la práctica de actividad física

El modelo del PAP caracteriza en siete etapas el cambio cognitivo-conductual desde un estilo de vida de riesgo (etapa 1) hasta uno de protección (etapa 7). Las etapas del modelo son acumulativas, es decir que una persona que se encuentre en una etapa superior reúne las condiciones de las etapas inferiores (19). Las etapas que componen el modelo son: 1) no conciencia del problema; 2) conciencia del problema, pero no compromiso personal; 3) decidir qué hacer; 4) decidir no actuar; 5) planear actuar, pero no se ha actuado; 6) actuar; y 7) mantenimiento de la acción (20).

Con el fin de desarrollar la adaptación del modelo para la explicación de la actividad física, se tomaron tres factores psicológicos, y se sopeso el valor de cada uno dentro de las siete etapas. Los tres factores psicológicos con los que se trabajó fueron cogniciones, emociones y motivaciones, los cuales a su vez presentan sub-divisiones. Para el factor de la cognición se toman en consideración dos aspectos, la información y las creencias evaluativas. La información se divide en información de eventos (memoria autobiográfica -memoria episódica-) e información de hechos (memoria no autobiográfica memoria semántica-) (21), mientras que las creencias evaluativas se divide en susceptibilidad percibida y severidad percibida. El factor de emocional lo componen los sentimientos derivados de la experiencia con la actividad física, que se representan con adjetivos bipolares como amor-odio, gusto-disgusto, admiración-desprecio y otros que denotan sentimientos de tipo favorable o desfavorable.

Por su parte, de la psicología del deporte se toma en consideración el amplio énfasis e investigación que se ha desarrollado sobre las condiciones motivacionales bajo las cuales se desarrolla la práctica de actividad física. En este sentido, se ha abordado la adherencia a la práctica de actividad física, bajo la explicación de las motivaciones que se presentan en la no práctica, 
práctica y mantenimiento de la actividad física (22-25). En este factor se vislumbra la amplia influencia de las emociones sobre la práctica, así, las emociones se encuentran ligadas con gran fortaleza con la motivación.

Con base en estos tres factores se adapta el modelo psicológico para la actividad física, tomando la información de cada factor y se aplica a las siete etapas del modelo. Luego de crear el modelo de caracterización psicológica de la práctica de actividad física, surgió la necesidad de comprobarlo estadísticamente, para lo cual se procedió a buscar un instrumento psicométrico de medición para realizar la comprobación del constructo, o en su defecto, optar por la creación de este.

\section{MÉTODOS}

Puesto que no se encontró un instrumento psicométrico que reuniera las características necesarias para el estudio fue necesario crear uno. Se partió entonces de la creación de las escalas y sub-escalas que conformaran el cuestionario, las cuales quedaron diseñadas como se ilustra en el Cuadro 1.

Cuadro 1. Conformación de escalas y sub-escalas

\begin{tabular}{|c|c|c|}
\hline Escalas & Sub-Escalas & Unidades de Análisis \\
\hline \multirow{2}{*}{ Factor Cognitivo } & Información Básica & $\begin{array}{l}\text { Información de Eventos } \\
\text { Información de Hechos }\end{array}$ \\
\hline & Creencias Evaluativos & $\begin{array}{c}\text { Susceptibilidad Percibida } \\
\text { Severidad Percibida }\end{array}$ \\
\hline \multirow{3}{*}{$\begin{array}{l}\text { Factor } \\
\text { Motivacional y } \\
\text { Emocional }\end{array}$} & Motivación de Inicio & $\begin{array}{c}\text { Razones de Práctica } \\
\text { Razones de no práctica }\end{array}$ \\
\hline & $\begin{array}{l}\text { Motivación de } \\
\text { Mantenimiento }\end{array}$ & $\begin{array}{l}\text { Diseño de Programas } \\
\text { Metodología de Práctica } \\
\text { Prevención de Recaídas }\end{array}$ \\
\hline & Emociones & \\
\hline
\end{tabular}

Luego de establecer las escalas y sub-escalas se creó un banco de 78 ítems que fueron validados por tres expertos que calificaron la pertinencia, claridad y relevancia. Posteriormente se desarrollaron tres pilotajes en los que se tuvo en cuenta homogeneidad y poder discriminativo de los ítems. Se propuso una escala Likert de cuatro opciones, en donde 1 equivale a muy en desacuerdo, 2 desacuerdo, 3 de acuerdo, y 4 muy de acuerdo.

Finalmente se realizó una aplicación con una muestra de 5,1 K (donde K es el número de reactivos total del instrumento). Se analizó la homogeneidad 
del instrumento por medio del $\alpha$ de Cronbach, partiendo de la conformación general, y tomando los componentes por separado (escalas y sub-escalas).

La homogeneidad del instrumento fue de 0,69 , mientras que las escalas "Factor Cognitivo" y "Factor Motivacional y Emocional" tuvieron un coeficiente de homogeneidad de 0,61 y 0,87 respectivamente. Por su parte la subescala Información Básica tuvo un coeficiente de 0,68, Creencias Evaluativas 0,62, Motivación de Inicio 0,78, Motivación de Mantenimiento 0,78, y Emoción 0,69.

Para la estimación de la validez se partió de un análisis factorial que se realizó mediante el método de componentes principales con rotación ortogonal tipo Varimax teniendo en cuenta las saturaciones de los reactivos por encima de 0,30. En cada uno de los factores se realizó un análisis de contenido con relación al constructo teórico de forma que se pudiera establecer la composición final de los factores. Se encontraron tres componentes principales, el primer componente se caracteriza por la presencia de reactivos de motivación y de emoción, el segundo contiene reactivos de creencias evaluativas y el tercer componente tiene en su mayoría reactivos de información. Estos tres componentes dan razón del 35,6 \% de varianza explicada.

Luego de la construcción del instrumento, se realizó la aplicación en estudiantes de la Universidad Nacional de Colombia. Para computar las puntuaciones de los individuos y del grupo poblacional para ubicarlos dentro de cada una de las etapas del PAP, se creó un protocolo de calificación y clasificación basado en el algoritmo teórico que aparece en la Figura 1. Para realizar la clasificación dentro de las etapas se promediaron los puntajes de cada sub-escala y se clasificaron como bajo, medio, o alto, según la escala Likert. En las sub-escalas de alto y bajo, la calificación de bajo se encuentra entre 0 y 2,5, y alto entre 2,6 y 5; mientras que en la sub-escala de creencias evaluativas, bajo se encuentra entre 0 y 1, medio entre 1,1 y 2 , y alto entre 2,1 y 5. 


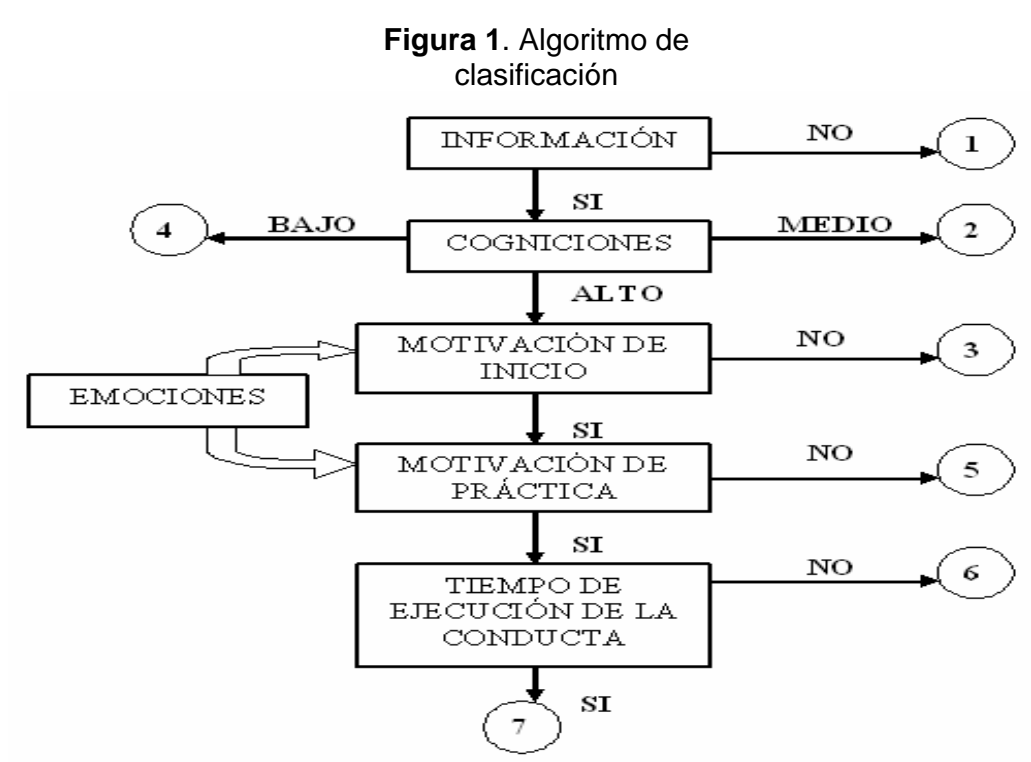

Los participantes en el estudio fueron estudiantes de la Universidad Nacional de Colombia con edades comprendidas entre 16 y 24 años, hombres y mujeres. El proceso de selección se hizo a partir de un muestreo aleatorio no probabilístico compuesto por 225 participantes. Para la aplicación del instrumento psicométrico se tomaron en cuenta las variables de edad, semestre cursado y carrera en la que se encontraba.

\section{RESULTADOS}

Para realizar la descripción de los resultados obtenidos a partir de la calificación de las pruebas de los estudiantes, se partió desde tres análisis: las puntuaciones globales de la población, las puntuaciones con relación a las facultades académicas, y las puntuaciones por rangos de edad.

Puntuación global de la muestra

El primer parámetro de medida es la calificación de la población en la prueba y su ubicación en las etapas del PAP. En la Tabla 1 se calculan las puntuaciones de la población en cada uno de los criterios de análisis, para luego establecer las relaciones de los factores psicológicos y la ubicación en las etapas del PAP. 
REVISTA DE SALUD PUBLICA • Volumen 8 (Sup. 2), Noviembre 2006

Tabla 1. Puntajes Globales de la Población Estudiantil

\begin{tabular}{cccc}
\hline $\begin{array}{c}\text { Criterio de } \\
\text { Análisis }\end{array}$ & Sub-escala & Media Ajustada $^{\text {a }}$ & Valoración \\
\hline 1 & Información & 2,30 & Descartada \\
2 & Creencias Evaluativos & 2,72 & Alto \\
3 & Ítem 27 & 3,04 & Alto \\
4 & Motivación de Inicio & 2,56 & Alto \\
\hline 5 & Emoción & 2,33 & Bajo \\
\hline 6 & Motivación de Mantenimiento & Emoción & Bajo \\
\hline
\end{tabular}

${ }^{a}$ Existen reactivos que puntúan de forma negativa, y otros de forma positiva

En esta instancia es necesario resaltar que la sub-escala de Información Básica no se comportó de forma apropiada en el análisis psicométrico. La sub-escala no presenta un discernimiento adecuado en la población, pues la mayoría de las personas obtuvieron puntajes muy bajos. Según la teoría esta situación no es muy ceñida a la realidad, ya que la población tiene un nivel intelectual alto, presentando asimismo la información necesaria para sobrepasar la Etapa 1 del PAP. Por tal razón, se revisaron los reactivos que componen la prueba, y se encontró un problema en su formulación: hay una desviación de su propósito de medición. Se decidió no contar con esta sub-escala que en realidad no resulta muy relevante en el trabajo descriptivo de esta investigación.

\section{Factor Cognitivo}

Este factor está compuesto por la Información Básica y las Creencias Evaluativas. La sub-escala de Información como se mencionó, no se tuvo en cuenta. La sub-escala de Creencias Evaluativas tiene un comportamiento adecuado y esperado. Presenta un puntaje alto superando el límite teórico propuesto para ubicarse dentro de las etapas superiores a la Etapa 2.

El criterio 3 (ítem 27) se compuso para detectar el sesgo optimista - no realista. El puntaje de la muestra es alto, demostrando que no existe una tendencia hacia la Etapa 4 del PAP. Es necesario determinar las puntuaciones en los factores siguientes para conocer la ubicación de la población en las etapas del PAP.

\section{Factor Motivacional y Emocional}

El factor de motivación se divide en dos sub-escalas: Motivación de inicio y motivación de mantenimiento. En el protocolo propuesto cada una de estas sub-escalas se promedia con el Factor Emoción para obtener el puntaje de 
ubicación. El puntaje obtenido por la muestra sobrepasa el umbral propuesto, analizando así el siguiente criterio.

En la sub-escala Motivación de Mantenimiento, el resultado está por debajo del umbral. En este sentido, la población objetivo de la investigación se encuentra en la Etapa 5 del PAP. Existe el criterio 6 (item 7) que detecta la conducta de la práctica regular de actividad física. Este reactivo mostró un comportamiento muy particular, ya que de la misma forma que el resto de ítems de la escala, no supera el umbral de clasificación para ubicarse en la Etapa 7.

\section{Mediciones complementarias}

Para realizar una revisión complementaria de las características poblacionales, se tomaron en cuentas otras variables, como edad, semestre cursado y Facultad a la que pertenecía el individuo. En cuanto a la edad, se dividió el grupo en personas menores y mayores de 20 años. De igual forma, en cuanto al semestre de estudio se dividió el grupo en personas que cursaban semestres inferiores o mayores de quinto.

Se calificó la prueba en cada grupo poblacional y se ubicó a la población dentro de las etapas 3, 5 y 6, puesto que las otras etapas no fueron registradas por ningún grupo poblacional. Los resultados arrojados permiten encontrar perfiles de riesgo y perfiles favorables para la promoción de la actividad física. En cuanto a los perfiles de riesgo se encuentran las personas mayores de 20 años, que cursan semestres superiores a quinto en las carreras de Agronomía, Ciencias Humanas o Derecho y Ciencias Políticas. En contraparte, las personas que tienen menos de 20 años, que se encuentran en semestres inferiores a quinto en las carreras de Odontología y Veterinaria, tienen grandes posibilidades de desarrollar un estilo de vida activo.

\section{DISCUSIÓN}

El objetivo central de la presente investigación fue adaptar un modelo teórico para la comprensión de la adquisición de estilos de vida activos, basado en el modelo del Proceso de Adopción de Precauciones (PAP). Con la construcción de este modelo se buscó dar cuenta del proceso de cambio cognitivoconductual, a través de las siete etapas del PAP. Se complementó el modelo a través de la caracterización de tres factores psicológicos básicos: el Factor Cognitivo, el Factor Motivacional y el Factor Emocional. Dicho constructo teórico se representó en un instrumento psicométrico que funcionara como 
herramienta metodológica para la descripción del estado y proceso cognitivoconductual en la población objetivo.

El instrumento tuvo un comportamiento apropiado, presentando niveles adecuados de homogeneidad y validez, pero la sub-escala de "información" no funcionó efectivamente debido a las características de la población. Esta escala se refiere a conocimientos generales sobre la actividad física y sus beneficios para la salud, así como en el sedentarismo y sus consecuencias.

La población objetivo de la investigación en la que se aplicó el instrumento posee un nivel intelectual alto, lo que implica un conocimiento amplio sobre los alcances de la actividad física y los efectos del sedentarismo. Este conocimiento se reflejó en la baja discriminación de los reactivos y de la subescala en general. Sin embargo, esta escala no debería producir una discriminación alta ya que según lo propuesto en principio, la población objetivo presentaba un conocimiento completo sobre la actividad física, pero no una práctica regular de ésta. Aunque se esperaría que la sub-escala no funcionara correctamente, no se tuvo en cuenta al principio de la medición.

En general los resultados obtenidos mediante la aplicación del instrumento se pueden clasificar en dos grupos de datos: la puntuación global y las mediciones complementarias.

Puntuación global

De forma global es posible aseverar que el factor cognitivo tuvo una puntuación muy estable entre toda lo población, ya que no se encontraron grandes diferencias entre los grupos. En este sentido, se puede afirmar que en la población existe una alta conciencia sobre los beneficios que conlleva tener un estilo de vida activo, y los riesgos para la salud de manejar un estilo de vida sedentario.

Por su parte, los factores psicológicos que más influencia tuvieron en el análisis de la población fueron el factor motivacional y el emocional, ya que funcionaron como filtro entre los grupos de la población. Estos factores fueron los más importantes en el análisis de la práctica de actividad física para la población, ya que denotan las falencias que tienen los estudiantes para iniciar la práctica de actividad física.

Mediciones complementarias 
Se pueden vislumbrar dos grupos poblacionales a partir de la aplicación del instrumento. El primer grupo de menores de 20 años y estudiantes de primeros semestres, y otro grupo de estudiantes mayores de 20 años y estudiantes de semestres superiores. El primer grupo se ubica en la etapa 5 del PAP (el mismo de la población general), mientras que el segundo grupo está en la etapa 3 del modelo. La etapa 3 se caracteriza porque las personas tienen conocimiento sobre la actividad física y sus valoraciones sobre ésta son positivas, pero no se han decidido a practicarla. Así se encuentra que a medida que los estudiantes adquieren mayor edad, van disminuyendo la práctica de actividad física, es decir que presentan estilos de vida sedentarios. De esta manera, a medida que los estudiantes permanecen en la universidad, disminuyen la práctica de actividad física.

En general el constructo teórico tiene una fuerte base experimental, al adaptar las bases para realizar el diagnóstico psicológico sobre la práctica de actividad física de una forma adecuada, respaldada psicométricamente. De esta forma es posible considerar que se cumplió con el objetivo principal de la investigación puesto que se encontró un punto de partida firme para la explicación de los procesos psicológicos inherentes en la práctica de actividad física. Aunque esta investigación no ahonda en aspectos tales como intervención, sirve como base conceptual para posteriores análisis o propuestas de intervención en el área de la promoción de la actividad física.

En cuanto al instrumento, es necesario ajustar la escala de información con relación a los requerimientos y a las características poblacionales para tener una medida más clara y precisa de la etapa 1 del PAP. También es importante aplicar el instrumento y estandarizarlo en otros grupos poblaciones como empresas, colegios o diferentes comunidades

\section{REFERENCIAS}

1. Organización Panamericana de la Salud -OPS. La salud de los adolescentes y jóvenes en las Américas: un compromiso con el futuro. Washington: OPS; 1985.

2. Capdevila L. Actividad física y estilo de vida saludable. Barcelona: Grup Artympres S.A.; 2000.

3. National Center for Chronic Disease Prevention and Health Promotion-CDC. Promoting physical activity. Journal of Preventive Medicine 2002; 22: 73102.

4. Jodra P. Deporte y salud: la realidad psicosociológica. Barcelona: Oikos Tau Ediciones; 1994. 
5. McInnis K, Franklin B, Rippe J. Counseling for physical activity in overweight and obese patients. American academy of family physicians 2003; 67: 12491256.

6. Fletcher G, Balady G, Amsterdam E, Chaitman B, Eckel R, Fleg J, et al. Exercise standards for testing and training. AHA scientific statement 2001; 16941740.

7. Story M, Neumark-Sztainer D, Sherwood N, Stang J, Murray D. Dieting status and its relationship to eating and physical activity behaviors in a representative sample of US adolescents. Journal of American Dietetic Association 1998; 98: 1127-1134.

8. Bryn A. Prevention research in eating disorders: theory and new directions. Psychological medicine 2000; 30: 1249-1262.

9. Dalle R. School-based prevention programs for eating disorders. Dis Manage Health Outcomes 2003; 11: 579-593.

10. Jakicic J, Clark C, Coleman E, Donnelly J, Foreyt J, Melanson E, et al. Appropriate intervention strategies for weight loss and prevention or weight regain for adults. Medicine and science in sports and exercise 2001; 33: 21452156.

11. Gómez M, Izquierdo M, De Paz F, González F. Influencia del sedentarismo en las desviaciones raquídeas de la población escolar de león. Revista Internacional Médica Científica de Actividad Física y Deporte 2003; 8: 215-249.

12. Koltyn K. The thermogenic hypothesis. Health psychology and behavioral medicine 1997; 3: 181-192.

13. Hoffmann P. The endorphin hypothesis. Health psychology and behavioral medicine 1997; 3: 193-202.

14. Dirección de Bienestar Universitario. 15 años de un nuevo modelo de bienestar universitario 1984-1999. Espacio abierto 1999; 6: 12-30.

15. Blasco T. Asesoramiento psicológico en programas de ejercicio. En: T. Blasco eds. Psicología del deporte. Barcelona: Síntesis Psicológica; 1997.

16. Fahrenwald N, Noble S. Application of the transtheoretical model of change to the physical activity behaviors of WIC mothers. Public health nursing 2003; 20: 307-317.

17. Flórez L. El proceso psicológico de la promoción y la prevención. Avances en psicología clínica latinoamericana 2000; 18: 13-22.

18. Flórez L. El proceso de adopción de precauciones en la promoción de la salud. Revista Argentina de Clínica Psicológica 2002; XI (1), 23-33.

19. Weinstein N. The Precaution Adoption Process. Health Psychology 1988; 7: 35 47.

20. Weinstein N, Lyon J, Sandman P, Cuite C. Experimental evidence for stages of health behavior change: The precaution adoption process model applied to home radon testing. Health psychology 1998; 17: 445 - 453.

21. Montañés P. Memoria y olvido. Neurociencias en Colombia 1998; 6: 31-45.

22. Cantón E. Motivación y su aplicación en el deporte. Valencia: Promolibro; 1999.

23. Roberts G. Motivación en el deporte y el ejercicio. Bilbao: Desclée de Brouwer; 1995. 
24. Weinberg R. Fundamentos de psicología del deporte y el ejercicio. Barcelona: Ariel Psicología; 1996.

25. Sánchez F. La actividad física orientada hacia la salud. Madrid: Biblioteca Nueva; 1996. 
